\title{
Bruno Latour y el estudio de lo social: construcción y actuación en red*
}

\author{
Bruno Latour and social studies: joint development and \\ intervention
}

Alba Lucia Cruz Castillo

\begin{abstract}
Resumen
El presente artículo hace una reflexión acerca de los aportes de Bruno Latour a las ciencias sociales, en especial al proceso de interpretación de "lo social" en el desarrollo de la investigación social; desde este lugar se retomaran dos cuestionamientos importantes, el primero, la forma tradicional de bordar lo social y sus implicaciones; en segundo lugar, las interpretaciones alrededor de su quinta incertidumbre y el papel de los científicos sociales; en tal sentido el artículo ilustra los cuestionamientos fundamentales que Latour se hace sobre los métodos de acercamiento a la realidad y la manera de procesar la información en la investigación social, llegando a la conclusión que allí es necesario problematizar la idea misma de 'construcción social' en la medida en que se convierte en otro 'apelativo funcional' que no supondría la necesidad de dar cuenta de las prácticas concretas.
\end{abstract}

\section{Palabras claves}

Social, investigación social, agencia.

\section{Códigos de clasificación JEL: Y30}

\begin{abstract}
This article presents a reflection on the contributions of Bruno Latour to the social sciences, especially the process of interpreting "the social" in the development of social research, from this place was rediscovered two important questions, the first form embroider traditional social and its implications, and second interpretations about its fifth uncertainty and the role of social scientists. The current bet is to seek results from research experiences a theoretical solution to the main question that drives us to the researchers and social actors "and for what we investigate".
\end{abstract}

\section{Keywords}

Social, social research, agency.

* Este artículo es resultado de la revisión teórica elaborada para la investigación denominada "Políticas, discursos y prácticas sobre la memoria, la reparación y la paz con adolescentes jóvenes víctimas del conflicto: reflexiones para la intervención profesional de trabajo social", desarrollada en el Centro de Estudios en Desarrollo y Territorio de la Universidad de La Salle.

1 Magíster en Estudios de Desarrollo, estudios doctorales en Antropología Social y Cultural, docente investigadora de la Universidad de La Salle, Bogotá, Colombia. Correo electrónico: alcruz@unisalle.edu.co; albaluciacruz@ yahoo.es 


\section{Contextualización de la apuesta de Latour}

Latour ${ }^{2}$ promueve, recurriendo a Tarde a otra sociología, al recuperar algunas discusiones expuestas por este en el campo del esparcimiento, posteridad y actividad cultural; de igual modo, alaga la relevancia que Tarde le asignó a la geografía política, así como su esfuerzo por establecer relaciones entre personas y grupos sociales, mostrando que los fenómenos de interacción social y reconocimiento mutuo se encuentran en el corazón de los colectivos. Latour recurre a la explicación de lo colectivo desde los ensamblajes o agregaciones y los transcursos de las personas, elaboradas por los mediadores con los que se encuentra en su camino; esta interacción concibe a los sujetos actores-red, lo cual hace referencia a explicar las interacciones desde las relaciones globales, locales y los sitios o relaciones situadas; lo que Latour argumenta en tres movimientos, que son necesarios para comprender esta relación; el primer movimiento refiere a la necesidad de cambiar de escala interpretativa sobre la realidad, con la intención de desanclar la explicación de los fenómenos sociales en conceptos determinadamente estructurantes; el segundo refiere a la necesidad de valorar el encuentro cara a cara en lo cotidiano de los sujetos; el tercer movimiento apunta a conectar elementos heterogéneos socialmente de otra manera a como se han entrelazado tradicionalmente; Latour a través de estos movimientos argumenta que no existe una cosa ni una propiedad específica que se pueda denominar "social", sosteniendo que las explicaciones tradicionales de lo "social" se han centrado en las manifestaciones de ello, más no en ello; por lo tanto critica la ontología de la sociología tradicional y propone un modelo que redefina lo social como resultado de interacciones concretas y materiales entre elementos que son en sí no sociales, refiriéndose al abandono de una teoría tradicional en la forma de explicar lo que no se conoce en su totalidad.

De la mano de John Law y Michael Callon, ha desarrollado la teoría del Actor-red que surge en los años ochenta como un enfoque provocativo, en la que propone el estudio de ensamblajes que involucran aspectos heterogéneos (legales, tecnológicos, organizativos, políticos, entre otros), donde los elementos deben describirse en su especificidad, y a la vez en su asociación conjunta que permiten las acción y la función compleja; de ahí se deviene el concepto de "agencia" para referirse a la capacidad de los "no-humanos" (máquinas, artefactos, entre otros) que pueden ser indispensables en el ensamblaje y a sus propiedades sustanciales, más allá de su rol en las redes. Latour en su indagación de los errores de la sociología tradicional expone como uno de los más relevantes la determinación de los fenómenos sociales a explicaciones conocidas, en tal sentido, la acción humana encuentra su mayor explicación en términos impersonales devenidos de fuerzas o estructuras sociales, en conocimientos de acción intencional; para contrarrestar esto Latour se propone utilizar un interlenguaje, es decir, términos flexibles que se desarrollan en un plano

2 Filósofo, sociólogo de la ciencia y antropólogo, francés. 
donde se rastrean conexiones a través de varios marcos de referencia; allí lo social es un "movimiento" de reasociaciones y ensamblado, en el que no hay nada establecido que pueda usarse como punto de partida incontrovertible.

Las huellas de lo social deben ser seguidas a través de los cambios sutiles "producidos al reconectar recursos no sociales" (Latour, 2001, p. 59), esto se presenta como un intento de dilucidar fenómenos muy complejos desde un enfoque radicalmente distinto, lo que implicaría dejar de lado el uso de la palabra "social" por la de "asociación", la cual daría cuenta de las múltiples conexiones que subyacen en los fenómenos, entre los elementos ontológicos heterogéneos que coparticipan en la construcción de todo hecho tecnocientífico. La teoría del Actor-red investiga todos estos vínculos sociales, desde la comprensión de sus entramados que pueden ser investigados empíricamente bajo el principio de la indeterminación radical respecto de si se suscriben como actores o actantes (término que acoge la teoría), que interactúan y forman nodos de los que se compone la red socio-técnica a la cual pertenecen.

La sociedad es en esta línea el resultado de asociaciones que dan lugar a colectivos de actores humanos y no humanos que conviven en otros colectivos de manera no siempre armónica, y entre los cuales se dan diversos mecanismos de interacción, donde no existen definiciones prestablecidas sobre quiénes son los actores, por cuáles principios se dirigen o cuáles son sus metas y objetivos, esto probablemente sea el resultado de investigar las dinámicas propias de los colectivos, pero no como resultado de premisas de estudio.

Bloor (1976) argumenta que la sociología y algunas disciplinas sociales han estado dispuestas a limitar su preocupación por la ciencia a su marco institucional y a factores externos, lo cual deja de lado tocar en realidad la esencia del conocimiento que lo social crea, en vez de definir el conocimiento como una esfera de verdad lo ha de definir como aquellas creencias que la gente sostiene confiadamente y mediante las cuales viven, en este punto este sociólogo al igual que Tarde y Latour ponen el conocimiento de lo social en la esfera de la complejidad y de allí se ocupan de las ideas que fundamentan el mundo del mundo mismo. A este modo particular de sociología Callon (1995) le llamaría sociología de la traducción, la que se pretende mostrar como un marco apropiado para el estudio del papel que desempeñan la ciencia y la tecnología en la estructuración de las relaciones de poder en la sociedad, que obedecen a una compleja red de interacciones en que la sociedad y la naturaleza se entrelazan.

Para la teoría del Actor-red podría presentarse como una gran dificultad la creencia que tanto humanos como no humanos tienen voz, esta concepción plantea algunos problemas al comprender los ámbitos de los reglamentos jurídicos, donde en efecto los derechos de los objetos frente a los sujetos, no son correlacionales, habría 
aquí un conflicto de fondo y es la noción de que los no-humanos están en pie de igualdad con los humanos, de igual forma la teoría presenta algunos hoyos al tratar de designar responsabilidades frente a las acciones en la esfera del contrato social; pero que sin embargo, no le restan importancia a su propuesta de desmitificar la ciencia y lo "social". Latour (1983) en su ensayo "Dadme un laboratorio y moveré el mundo", mostraría que la mayoría de científicos no trabajan con respuestas prescriptivas, que por lo contrario, trabajan sobre acciones a posteriori, en el mismo caso funciona con lo social, de igual forma sobre esta metáfora retoma el tema del afuera y el exterior; aludiendo que lo social debe partir del adentro hacia afuera y no al contrario; en tal sentido esta dicotomía cobraría otro sentido, el de la interacción y no la determinación.

\section{Quinta incertidumbre}

La quinta fuente de incertidumbre en la apuesta de Latour tiene la intencionalidad de recoger el proceso reflexivo que se ha propuesto en las cuatro anteriores apuestas; en esta ocasión la incertidumbre se presenta a manera de proposición política, en ella expone la importancia de la narrativa como escena estética y práctica profesional; en esta quinta incertidumbre Latour expone su legado de Derrida, frente a la independencia de la construcción narrativa, y de la responsabilidad que cada texto tiene cuando se expone como único o como particular al mundo; de igual forma pongo en escena la tensión entre la relevancia de la crítica del autor y las voces de los informantes, y la manera en que algunos textos científicos exaltan la primera y olvida o invisibilizan la segunda.

Para Latour, las buenas narrativas nunca se autoproclaman como solo historias, por el contrario, hay una liberación de la necesidad de ser objetivas, el relato social debe estar susceptible a la falla, "si lo social es una búsqueda entonces puede ser recuperado. Si es un ensamblado, entonces puede ser reemsamblado" (Latour, p. 128); aquí el autor retomaría elementos de Jhon Law, en los que hay un lugar político en lo que se elabora como informes de investigación, aludiendo que la visión del mundo que se plasma en un informe de investigación normalmente es normativa, porque de esta manera hemos aprendido a investigar; Law reflexiona al igual que Latour sobre las prácticas investigativas, basándose en múltiples ejemplos y resúmenes de investigación, donde los objetos son mostrados como certezas y no como flujos de conocimiento; en lo que se acercarían estos dos autores es precisamente en la denominación de la complejidad de los objetos, esta nueva ola de pensadores intenta ir más allá de pensar la premisa que las realidades están inmersas en los métodos.

La función del investigador desde la teoría del Actor-red es el despliegue, que el informe permita visualizar las múltiples mediaciones de los actores, y logre un mapeo de la complejidad de la realidad, este es el verdadero sentido del guion, no 
solamente es describir, o una muestra de alguna fuerza relevadora de certezas frente a lo social; en este guion además el investigador debe ser consciente del esquema de mediaciones necesarias para la descripción, lo cual partiría de las asociaciones. Esta cartografía de lo social permite desprenderse de los planos analíticos por niveles interpretativos macro, meso y micro, en cambio de ello, relocaliza lo global y redistribuye lo local, dado que la naturaleza de lo que denominamos social parte de las asociaciones. El punto de observación y relato desde donde parte el investigador, lejos de ser un panóptico es oligóptico que produce visiones en detalle y de detalles, pero parciales; esto es ver muy poco, pero ver muy bien.

Esta quinta incertidumbre, que otros autores la abordarían como órbita, representa los vínculos y elementos vinculantes, consiste en mantener en movimiento los cuatro anteriores elementos u órbitas y a la vez fundirlos, dotando a los textos científicos de la capacidad de reflejar la comprensión de los acontecimientos que los generan y las razones de ello, de esta manera se da unicidad a la historia social de las ciencias; como resultado o provocación de desmontar la visión de la ciencia que parte de la división entre lo interno y lo externo, en un modelo interpretativo que Latour denomina modelo rosácea que da cuenta de cinco momentos fundamentales: 1) movilización del mundo, 2) automización, 3) alianzas, 4) representación pública y, 5) vínculos y elementos vinculantes.

La exposición de estas conexiones entre actores y sus redes es lo que debe plasmar un informe final, con el cual en definitiva se apuesta a formar nuevos entramados de objetos y seres humanos; para Latourt y Woolgar la construcción social de la ciencia contiene factores, dimensiones heterogéneos que no se pueden restringir a la noción de dato, en cambio estarían las nociones de inscripción literaria y de inscriptores que dan cuenta de las narrativas investigativas y de su gama de trazos que relatan la complejidad de los hechos sociales y su dimensión de factibilidad en el discurso de orden social, donde el investigador realiza un seguimiento a los pasos de los actores y traduce sus lenguajes, a la vez que comprende que los actores, individuos, colectivos, humanos y no humanos trabajan constantemente en la construcción de sus identidades a través del lenguaje.

De cierto modo, la obra de Latour devela el proceso de creación de un método y una forma de llevarlo a la narrativa, a través de la honestidad del relato y los alcances de la buena observación y reflexión etnográfica. La construcción social del conocimiento implica en esta propuesta reinterpretar lo social poniendo de evidente en principio dos grandes tensiones epistemológicas, la primera la concepción de lo social como algo dado y no como el proceso a través del cual se ha construido algo colectivamente donde se requiere de la colaboración de muchas redes y agencias humanas y no humanas; la segunda tensión entre el supuesto de que lo social se enmarca exclusivamente en trazos de poder y la propuesta latourciana de que lo que hay que resaltar en los procesos investigativos son los procesos colectivos que 
culminan en la producción de sólidos constructos a través de la movilización de materiales y "artesanías heterogéneas coordinadas"; lo que implica hablar el tema de las redes y los agentes que dan vida a lo social.

En este sentido el tipo de agente que aborda Latour es un "constructor" que comparte su agencia con múltiples actores sobre los cuales no tiene inferencia ni control; en términos metodológicos el autor propone desde la Sociología de las Asociaciones estructurar en ejercicio de comprensión y de develación de dichas asociaciones, su entramado de actores y la naturaleza de las agencias implícitas allí, básicamente en tres pasos, primero una búsqueda de las controversias alrededor del caso de estudio, esto con el fin de no partir de concepciones prefiguradas de la realidad, para posteriormente rastrear la naturaleza de lo social.

Segundo, se identifica el modo en que circulan algunas agencias a través de mediadores e intermediarios, con la intención de apreciar el papel de los actores-red y el valor del uso de oligópticos. Tercero, se observan los límites de la investigación social y las estrategias para dar a conocer los resultados o hallazgos, donde juega un papel fundamental una epistemología política, referida a dónde y para quién se produce el informe y con qué intencionalidades.

Latour aborda el estudio de la práctica de los investigadores con una metodología antropológica, en el que pretende tratar a estos como un grupo social que se encuentra inmerso en redes técnico-económicas y socio-técnicas y, por lo tanto, sus postulados, en este texto brevemente expuestos, que proponen una reflexión de la naturaleza de emergencia de los hechos científicos y las redes en que estos se desarrollan, haciendo énfasis en los mecanismos de producción (construcción) como resultados de una conjunción de entidades humanas y no humanas, colectivas e individuales, definidas por los roles, la identidad y forma en medio de una disputa constante por la validez y la propia emergencia del conocimiento.

\section{Investigación como campo político}

Latour retomando a Platón reflexiona acerca del sentido del conocimiento y evoca su principal intencionalidad de ser distribuido para todos; en este sentido cuando el conocimiento se pone al servicio de los expertos pierde la posibilidad de ser explorado y a la vez su carácter político; en un segundo instante el autor propone que la segunda característica que puede recuperarse consiste en que la razón política no tiene posibilidad alguna de convertirse en objeto de estudio para ningún conocimiento profesional. Latour llama la atención en relación de lo que se necesita no es conocimiento como tal sino una muy concreta forma de atención a la totalidad de la comunidad por parte de la comunidad, es decir, son necesarias las condiciones para que este conocimiento llegue a quienes necesita que llegue. 
Latour critica un alejamiento de la práctica investigativa de lo humano, un nuevo tránsito para comprender la investigación desde otro lugar implica un ligero cambio en nuestra definición de ciencia y en nuestra definición de política. La investigación ha caído en una práctica que en palabras del autor "cortocircuita la política" y alude a una definición de ciencia (con $\mathrm{C}$ mayúscula) y es aquella cuya intención es ofrecer un sustituto para la discusión pública. "abolir las restricciones propias de la política ha sido siempre una rama política. Desde el principio, como vimos en el diálogo, la política había sido diseñada a la medida para este solo fin, y nunca ha dejado de usarse, en toda época de esa forma" (Latour, 2001, p. 309). Debido a que la Ciencia se aferra a esta premisa se vuelve inútil en su intencionalidad propia. Una segunda definición abordaría la ciencia como conocimiento de lo no humano, que en principio son entidades por fuera de la vida social y que poco a poco van siendo socializadas, a través de los ejercicios y puentes tendidos entre los laboratorios, las instituciones, constituyendo una fábrica con un repertorio donde se accede al conocimiento a través de la práctica.

Latour no pone en tela de juicio la relevancia de la ciencia con estas definiciones o que esta no sea objetiva, racional o positiva, lo que expone es que ni la ciencia ni la realidad son trascedentes e inmanentes, sino más bien al contrario, contingentes y cambiantes; incluso desde el mismo instante en que el investigador se ocupa de ver la realidad desde una óptica disciplinar, por encima de una historicidad que le configura. Lo real queda configurado por cuatro aspectos que están presentes a lo largo de toda la obra: epistemología, moral, política y psicología. Para comprender el campo complejo donde se desarrollan los procesos de investigación, el autor recomienda tener un especial cuidado en analizar las relaciones que le subyacen a la investigación y a las transformaciones que se dan en lo que propone llamar referencia circulante.

El autor hace especial énfasis en recordar que la ciencia es socialmente construida en la interacción entre científicos, y desde este punto de vista cada ida y venida, del laboratorio al ambiente, del laboratorio a conversatorio de pares, entre otros, lo que hace es alejar cada vez más la ciencia del entendimiento, en tanto este se logra mediante el acceso a la información obtenida del laboratorio y los datos, no de las propias fuentes. Al finalizar este proceso el científico concreta su desarrollo en un informe académico, informe que de igual manera se aleja del mundo real, sustituyéndolo, convirtiéndolo en un signo. El cuestionamiento es que tanto de lo que se reporta es lo que se vive y quienes están beneficiados por ello, sabiendo que cada intervención en la realidad la modifica y a su vez la habilita para ser comprendida desde una lógica y unos parámetros establecidos desde el mundo académico, que no forman parte del mundo natural de donde es el objeto real investigado.

La función del investigador no es en el fondo la comprensión de la ciencia, sino de la red de conexiones que se subyacen a lo que estamos abordando de la 
realidad; el distanciamiento entre el objeto y el sujeto es un fondo político, donde la investigación juega un papel fundamental en la comprensión de la naturaleza de los hechos; desde este punto de vista la epistemología política con la que debemos abordar una objetividad parte de realizar una apertura a los métodos y métodos de abordaje científico, para entender en las asociaciones que se tejen entre la naturaleza de las cosas, las cosas en sí y quienes la observan. "Latour ha propuesto reunir la actividad científica sobre la naturaleza en el juego normal de la sociedad y la acción política en la integración progresiva de un mundo común” (Arellano, 2003).

\section{Construcción social de la tecnología}

Este modelo teórico se ha constituido como uno de los modelos más relevantes en el estudio de la dinámica de la tecnología y de la Ciencia, al analizar lo que se encuentra en estas relaciones, se ubica una sociedad en acción, donde se plantea como necesario pensar la investigación como un proceso que pasa por la negociación social, tanto de quienes la lideran, como de los demás actores implicados allí; lo que se traduce en una deconstrucción de la visión lineal de la investigación; en este proceso de negociación social implícito en el conocimiento de un objeto que se focaliza para la investigación, se distinguen por lo menos cinco herramientas: primera, la existencia de grupos sociales, que presentan visiones de acuerdo con sus particularidades; segunda, el fenómeno de flexibilidad interpretativa con relación a los problemas y las soluciones; tercera, mecanismos de diálogo de los intereses de la comunidad y lo deseado o expresado por los científicos que regularmente se da por autoridad discursiva o ventaja técnica; cuarta, un marco tecnológico que enuncia y conceptualiza y ponen el conocimiento científico en un lugar determinado $\mathrm{y}$, finalmente, los actores incluidos o excluidos del marco tecnológico, que a la vez lo reproducen o conforman una fuerza opositora.

Latour, al igual que Callon tratan de explicar los mecanismos de poder de la ciencia y la tecnología y la manera en que los laboratorios reelaboran y relacionan los contextos sociales y naturales sobre los que actúan; esto presupone que la vida de los objetos de análisis en los laboratorios, o de las situaciones sociales al estudio de los científicos; producen cambios en la sociedad, por lo tanto es importante caracterizar y desde el análisis etnográfico desentrañar, el modo en que los científicos actúan, lo que ocurre en el interior del proceso de análisis y recolección de la información y, sobre todo, el análisis de los mecanismos que les permiten actuar sobre la sociedad en extensión, en este análisis se incluye la caracterización de estrategias, intereses y relaciones de poder intrincada a ellos. 


\section{Problemas en la investigación social que evidencia la puesta de Latour}

Es evidente que la propuesta de Latour, frente a lo que implica investigar y el juego de poder que hay en ello, deja entrever un debate contemporáneo alrededor de rol de la investigación social con respecto a la realidad que aborda; es un intento por mirar las cosas de otro modo. Enfatiza en que la acción no es lo que la gente hace, sino el hacer-hacer, lo que se logra con otros muchos, las oportunidades que el hecho produce y las circunstancias en que se produce; esto combinaría la interpretación de la acción social con tres cosas fundamentales: la definición de la acción y el dominio de esta, la localización de la acción, y la división entre lo humano y no humano que confluyen en la dinámica de la acción.

Con ello, el autor desea llamar la atención en relación con reflexionar acerca de los campos de poder que subyacen en el tratamiento de los problemas sociales como objetos de conocimiento; para alejarse del modo tradicional de concebir la ciencia hay que tratar de comenzar la totalidad de elementos que conjugan en ellos, elementos humanos y no humanos, y las interacciones que se realizan efectivamente. La práctica científica está colmada de una serie de transformaciones o traducciones que suceden de modo continuo, por tal motivo, en principio se puede suponer que el abordaje de las situaciones sociales necesita de un acompañamiento reflexivo de la sociología implícita en ello como una acción investigativa que está directamente vinculada a esta y no por fuera de ella.

Todo esto implica que la "realidad" no es algo dado o puesto, sino algo que se va transformando continuamente, hasta finalmente terminar siendo una cosa, una cosa del mundo, igualmente estos elementos que se llaman realidad han sido intervenidos desde una perspectiva determinada para denominarlos como tal, se trata de realizar de manera rigurosa una "etnografía del laboratorio", donde se hagan evidentes estas relaciones entre sujetos, objetos, o los lugares de sujeto y objeto que se ocupan en lugares, espacios y lecturas culturales y científicas específicas.

Hay varias razones por las cuales se puede decir que Latour propone una reacción epistémica a una ciencia enfrascada en sí misma, una ciencia capaz de estar en el mundo e interpretarlo, "Latour nos enseña que hay otro modo de acceso/relación con la realidad, distinto del que la Modernidad nos tiene acostumbrados" (Espuny, 2014, p. 8), esta nueva mirada para muchos es interpretada como nueva alianza, que pone en diálogo a la naturaleza con la ciencia, para lo cual en principio es necesario reconceptualizar los esquemas bajo los cuales hemos aprendido los procesos de acercamiento a la realidad, de esta forma crear marcos diferenciadores que den nuevos sentidos de los conceptos que nos enmarcan dentro de la lectura moderna de la forma de conocer, un ejemplo de ello es la reconfiguración de sujeto/objeto 
por humanos/no humanos, con la intención de reemplazar la distancia creada entre el sujeto que conoce y la cosa por conocer, que pone en el plano de conocimiento la relación pasiva y entre contrarios, por el contrario de una relación interactiva; "otro ejemplo muy utilizado y en relación con el concepto de comprensión, es el de traducción, que sugiere la idea de desplazamiento, deriva invención, mediación" (Espuny, 2014, pp. 9-10).

Con la propuesta de reconfigurar el método de conocer, Latour provoca un desafío al modelo lógico matemático de las ciencias naturales, que se piensa una realidad única no solo desde lo cuantitativo sino también lo cualitativo, de modo que en síntesis "se puede reunir la ciencia en una sola", mathesis universalis ${ }^{3}$, ciencia que en principio consta de igual manera de un lenguaje universal que se ajusta a la verdad, quizá una verdad ciega que se acomoda a un proyecto de modernidad como única forma de existir, de estructurar el mundo desde lo objetivo, un mundo material en donde el método tradicional se transforma en objeto, en donde existe la reversibilidad de los fenómenos como principio de duplicidad, cada fenómeno puede llegar a conocerse y ser certeros, este tipo de conocimiento se llama internalismo; la apuesta de la ciencia moderna es conocer la totalidad del fenómeno para controlarlo.

Las relaciones entre fenómenos, tiempo y espacio nos muestran que hay irreversibilidad, que hay fenómenos que no se repiten, que la realidad se mueve más por la probabilidad que por la certeza y que la realidad es construida, Latour centra su discurso en un proceso que evoca una ciencia práctica en acción, de acuerdo con (Prirogine \& Isabelle Stengers, 1994) implica modificar el estar de la ciencia:

el siglo XXI, a pesar de ser el momento de conciencia de las limitaciones de la ciencia natural y de la fuerte oposición de los saberes sociales (aunque seguramente a causa de esto), es también el momento culme de la omnipresencia de la ciencia. Es el siglo por excelencia de la mecanización de toda la realidad. El fin de este proceso es el progreso en el conocimiento. He aquí la clave de toda ciencia moderna: conocer significa saber manipular. Comprender significa interactuar. Estos son los dos conceptos - clave... el paso del deseo de conocimiento de la naturaleza al de la comprensión nos hace pensar de la alianza moderna, a la nueva alianza, de la ciencia moderna, a la no - moderna y de todo un modo de sestar en el mundo con base en el dominio a un nuevo modo de estar, como componentes de él (Prirogine \& Isabelle Stengers, 1994, pp. 24-27).

Desde estos postulados la nueva ciencia se caracterizaría por estudiar el devenir, en cambio de lo inmutable; en "donde la ciencia deviene con el resto de la realidad" (Espuny, 2014, p. 10), el discurso que antes era alejado de la naturaleza misma de los fenómenos pasa a ser un diálogo con la naturaleza, donde: ni la ciencia nos construye, ni construimos ciencia, sino que interactuamos desde los saberes, en los que no hay dominio de uno sobre el otro; estas relaciones darán como resultado una

3 Del griego matheseôs: ciencia o aprendizaje; latín universalis: universal, hace referencia a un hipotético modelo matemático previsto por Leibniz y Descartes. 
ciencia en proceso, que dialoga con una realidad en las mismas condiciones, expone entonces unas reglas sobre el método de conocer que se centran en: estudiar desde una ciencia en acción, estudiar los fenómenos en su devenir y transformaciones, el punto de partida de la argumentación no es la muestra causal de los fenómenos sino la representación de ellos en la relación con la naturaleza, donde se debe considerar la simetría entre lo humano y lo no humano, es decir, entre el sujeto, el mundo y su comprensión; cuando aparentemente se presente una acción científica irracional, la explicación de esta no es la fractura de una regla universal, sino en punto de vista del observador y su ángulo de interacción con el fenómeno y la red que lo construye $\mathrm{y}$, por último, antes de atribuir características hay que examinar las diversas formas, combinaciones y entrelazamientos que el fenómeno tiene.

Estas reglas ponen la cuestión además, en un nuevo tipo de análisis, uno que Latour llama conectivo, en el que la circularidad es la clave de comprensión del fenómeno, "según el diámetro de la circularidad, tendremos una mejor o peor comprensión, con base en sistemas más grandes, mayor número de interconexiones, referencias, riqueza y mayor probabilidad de comprensión"; el modelo de traducción, propuesto por Callon y Latour en 1981 (Latour, 2001, p. 111) advierte que esta circularidad puede contener una limitación en la mirada interna y externa de los fenómenos, es lo que lo lleva a plantear que la posición del observador cambia la mirada del fenómeno, en relación con ello propone hacer diferentes miradas desde los "bucles" (actividades enlazadas por redes) que los estudios necesitan considerar con "el fin de reconstruir la circulación de los hechos científicos" (2001, p. 121).

El modelo a manera de bucles (denominados: instrumentos, autonomía, alianzas, difusión pública y vínculos, nudos y conceptos) impulsa diferentes articulaciones que establecen relaciones múltiples, a lo que se llama traducción, entendiendo esta como el desplazamiento "que se verifica a través de actores cuya mediación indispensable para que ocurra una acción. En vez de una oposición rígida entre el contexto y contenido,...las cadenas de traducción se refieren al trabajo mediante el cual los actores modifican, desplazan y trasladan sus contrapuestos intereses" (Pineda \& Molero, 2012, p. 16).

El modelo de traducción llamado también en red, promueve la acción en la ciencia, mediante la interacción, integra de manera dialógica los elementos internos (contenido) y externos (contexto), con la intención de hacer viable la comunicabilidad como uno de los propósitos de la nueva ciencia; los bucles que interactúan en el proceso investigativo son representados por (Latour, 2001, p. 123): 


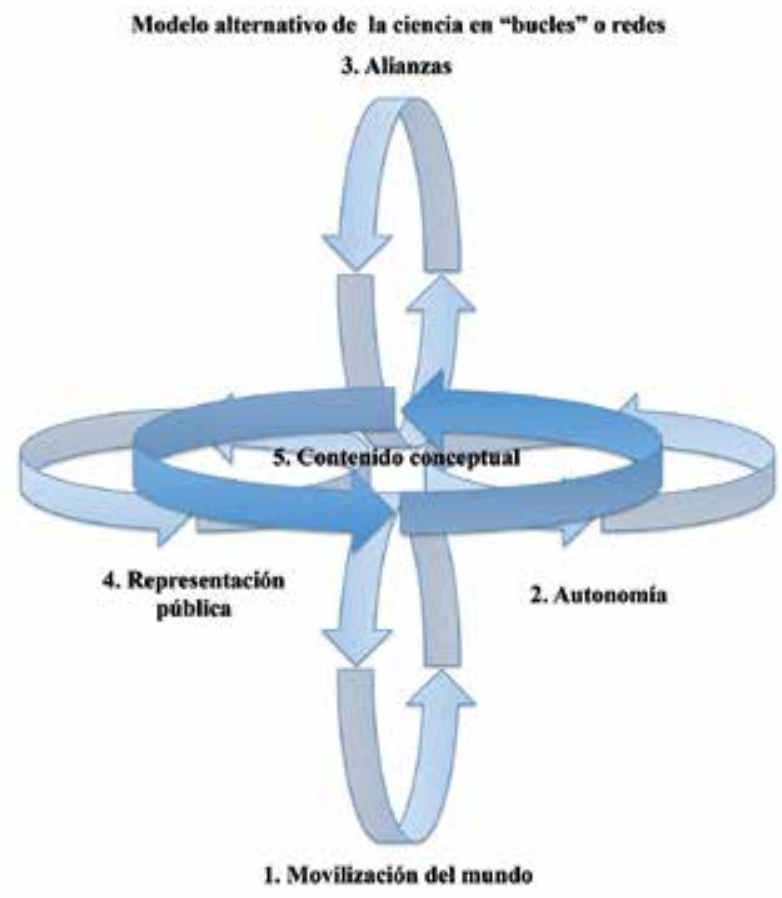

Figura 1. Modelo alternativo de la ciencia en bubles o redes. Fuente: Latour (2001).

La explicación que Latour asigna a este modelo es la siguiente: "el bucle 'movilización del mundo' comprende de manera muy general 'todos los medios por los cuales los no humanos quedan incluidos en el discurso de la CTI (ciencia, tecnología e innovación)"' (Latour, 2001, p. 128); el bucle de autonomía se refiere al cuerpo de sujetos que interpretan la realidad bajo el foco de las disciplinas o los saberes, nadie en su parecer puede especializarse sin un pequeño grupo de iguales que validen los saberes; el bucle de alianzas constituye la red mediante la cual se aplica y difunde el saber, en tal sentido las disciplinas tienen un rol fundamental en la existencia y perdurabilidad de este; finalmente, el bucle de la representación pública se refiere a los mecanismos de socialización en masa de los nuevos objetos de conocimiento; el modelo en su estructura presenta una fluida y constante interacción entre los bucles y aluden al constante movimiento de la realidad.

Como consideraciones finales se podría decir que los modelos de interpretación de la ciencia y el modo en que se investiga en el presente siglo aún mantienen una mirada cartesiana y kantiana sobre la cual no se ha discutido a profundidad, y sin mayores reflexiones se ha aceptado el planteamiento discursivo de objeto y sujeto en las ciencias, que pueden no serlo, ya que parte de lo que nos demuestra Latour es 
que el devenir de las interacciones y las formas en que ambos se configuran pueden ponerlos en lado u otro del análisis, así como en lugares compartidos frente a la misma interpretación de la acción social científica.

Un objeto científico-técnico es resultado de la mezcla de entidades humanas y naturales que actúan socialmente con las características de un actor situado entre dinámicas sociales y leyes naturales, a las posibilidades de ambos; aquí la noción de red ha sustituido de alguna manera a la de sistema y desde allí se interesa en delimitar y poner en relación elementos de la realidad o de una parte de esta sin una jerarquía precisa o lineal, considerándose que los elementos encuentran interacción por medio de circulación de información que se da en una esfera social donde se han solidificado en procesos de traducción socialmente construidos.

El llamado de Latour es a desmitificar el rol del científico y la práctica científica, al igual que el discurso científico como producto exclusivo de las élites; en su lugar aboga por realizar una interpretación más profunda de la práctica científica y el rol que juega en el posicionamiento de lo "científico" el discurso de quien investiga; al igual que las interrelaciones entre sujeto y objeto de conocimiento. En este sentido se acude a la existencia de una ontología variable, lo que significa que la noción de sujeto puede ser vista como la de un agente y la de objeto como no pasivo, pues en algún momento del proceso constructivo e interpretativo de la investigación, los humanos somos también objetos, somos quienes transformamos a través del quehacer científico y tecnológico y a la vez estamos permeados por la historia y los condicionamientos culturales bajo los cuales leemos la realidad.

\section{Referencias}

Arellano, H. A. (2003). La sociología de las ciencias y las técnicas de Bruno Latour y Michael Callon. En Escuelas sobre la tecnología. Universidad Autónoma Chapingo. México.

Bloor, D. (1976). Conocimiento e imaginario social. Barcelona: Gedisa (1998).

Callon, M. (1995). Algunos elementos para una sociología de la traducción: la domesticación de las vieiras y los pescadores en la bahía de Saint Brieuc. En Iranzo, J. M., Blanco, R., González, F. T., Torres, C., y Cotillo, A. (Eds.). Sociología de la ciencia y la tecnología (pp. 259-282). Madrid, España: CSIC.

Callon, M. (1999). Actor-network theory-the market test. The Sociological Review, 47(S1), 181-195.

De Castro, M. I. (2006). ¿Una epistemología sin sujeto? Opción, 22(51), 87-110.

Espuny, J. (2014). Bruno Latour, los estudios de la ciencia y la comprensión. A Parte Rei, 22, 1-16. 
Latour, B. (1989). La Science en action (p. 209). (Trad. Francia, París, La Découverte). (1 ${ }^{\mathrm{a}}$ ed. Americana, 1987).

Latour, B. (1995). Dadme un laboratorio y moveré el mundo. En Iranzo, J. M., Blanco, T., González, F. T., Torres, C., y Cotillo, A. (Eds). Sociología de la ciencia y la tecnología (pp. 237-257). Madrid, España: CSIC.

Latour, B. (2001). La esperanza de Pandora. Ensayos sobre la realidad de los estudios de la ciencia. Barcelona, España: Gedisa Editorial.

Latour, B. (2005). Reassembling the social. An Introduction to Actor-Network Theory. Oxford: Oxford University Press.

Law, J. (1999). Alter ANT: Complexity, naming and topology. En Law, J., y Hassard, J. (Eds.). Actor Network Theory and after (pp. 1-14). Oxford: Blackwell Publishers.

Law, J. (2004). After method. Mess in social Sciencie Research. London: Routledge.

Pineda, A., y Molero, L. (2012). Concepción semiótica de la tecnociencia en Bruno Latour. Apuntes para una comunicación pública.Enl@ce, Revista Venezolana de Información, Tecnología y Conocimiento, 9(3), 11-33.

Prigogine, I., y Stengers, I. (1994). La nueva alianza: metamorfosis de la ciencia. Madrid, España: Alianza Editorial. 